\title{
Cytochrome P450 Enzymes and Microbial Drug Preparation
}

\author{
Wan Feng Li \\ Changsha Medical University, Changsha, China, 410219 \\ 425680231@qq.com
}

Keywords: Cytochrome; P450 enzymes; Microbial Drug

\begin{abstract}
This paper aimed to introduce cytochrome P450 (CYP450) enzymes and illustrate the enzyme catalytic mechanisms. Notably, this paper had focused on the relation of CYP450 with microbial drug preparation; meanwhile, it had pointed out the challenges faced by the future development of P450 enzymes.
\end{abstract}

\section{Introduction}

Cytochrome P450 (CYP450) enzymes are the principle members of heme-thiolate protein superfamily, with heme being the prosthetic group. CYP450 enzymes are extensively involved in processes like xenobiotic degradation, which marks the focus of attention in current microbial pharmaceutics. Research on the relation of CYP450 enzymes with microbial drug preparation is of great value to promote the drug manufacturing efficiency and quality.

\section{CYP450 enzymes and their catalytic mechanisms}

CYP450 enzymes have numerous functions, which have high application superiority in terms of catalytic reaction. CYP450 enzymes can be divided into different types based on different criteria. Specifically, they can be classified into 4 types according to host source, as was shown in Table 1.

Table 1. Host source-based CYP450 enzyme classification

\begin{tabular}{ccccc}
\hline Host source & Animal & Plant & Bacterium & Fungus \\
\hline $\begin{array}{c}\text { P450 } \\
\text { enzyme }\end{array}$ & Animal & Plant P450 & Bacterial & Fungal \\
enzyme & P450 enzyme & P450 enzyme
\end{tabular}

Support from reducing chaperonin is indispensable in the realization of catalytic reaction when CYP450 enzymes are applied in the catalytic process. The mechanisms of action of P450 enzymes are different in different catalytic reactions; meanwhile, there is also difference in enzyme types (Table 2). Take hydroxylation as an example, the P450 enzyme in that reaction is hydroxylase. The enzyme catalytic mechanisms are shown as follows. Firstly, the substrate enters the enzyme active site. Secondly, the reducing chaperonin participates in the reaction, so that Fe3+ in heme can be reduced. Thirdly, the reduced substance reacts with oxygen to produce the compound. Fourthly, the reducing chaperonin takes part in the reaction again, and the compound obtains the electron and proton. Fifthly, the free radical is formed. Sixthly, the compound becomes the dehydrogenation catalyst to produce the hydroxylation product. Seventhly, the product separates from the P450 enzyme active site, and the catalysis is completed.

Table 2. Catalytic reaction-based CYP450 enzyme classification

\begin{tabular}{cccc}
\hline Type & Type 1 & Type 2 & Type 3 \\
\hline $\begin{array}{c}\text { P450 enzyme } \\
\text { classification }\end{array}$ & Epoxidase & Isomerase & Hydroxylase \\
\hline
\end{tabular}




\section{CYP450 enzymes and microbial drug preparation}

\section{The application of CYP450 enzymes in microbial drug preparation}

The application of CYP450 enzymes in microbial drug preparation was discussed from three aspects, namely, biosynthesis, enzyme modification and drug manufacturing.

\section{Biosynthesis}

CYP450 enzymes showed high application value in biosynthesis. The functions of enzyme in synthesis were shown in Table 3.

Table 3. The functions of CYP450 enzymes in biosynthesis

\begin{tabular}{ccc}
\hline $\begin{array}{c}\text { Functi } \\
\text { ons }\end{array}$ & Function 1 & Function 2 \\
\hline Clas & $\begin{array}{c}\text { Microbial secondary metabolite } \\
\text { sificati }\end{array}$ & Plant natural product synthesis \\
on & biosynthesis & \\
\hline
\end{tabular}

Function 1: Microbial secondary metabolite synthesis was the basis of disease treatment. Took penicillin as an example; the manufacturing of penicillin was the microbial secondary metabolite synthesis. There was certain difference in the synthetic route according to different drug categories. The details were shown in Table 4.

Table 4. Microbial secondary metabolite biosynthetic routes

\begin{tabular}{cccc}
\hline Route & Route 1 & Route 2 & Route 3 \\
\hline Category & Polyketide & $\begin{array}{c}\text { Non-ribosomal } \\
\text { peptide }\end{array}$ & NRPS-PKS \\
\hline
\end{tabular}

P450 enzymes were dominated by microbial enzymes in the above three synthetic routes, which had relatively single function and could only participate in a same category of reaction. Compared with microbial P450 enzymes, CYP450 enzymes had relatively more functions, more abundant catalytic types and more outstanding application value.

Function 2: CYP450 enzymes were also applied in plant natural product synthesis apart from animal. Research had indicated that plant secondary metabolite interacted with other substances after synthesizing the gene cluster, which could be manufactured into drug. CYP450 enzymes played irreplaceable roles in drug manufacturing process. Took artemisinin as an example, the synthetic process was shown below. Firstly, prepared the Artemisia annua and extracted CYP71AV1 from P450 enzymes. Secondly, to obtain the arteannuic acid through oxidation. The oxidation process was relatively complicated, which mainly included three major steps. 2 CYP88A were involved in the oxidation. Ent-kaurene could be initially gained after oxidation, which could finally lead to arteannuic acid after CYP450 enzymes catalysis [1].

\section{Enzyme modification}

The application value of CYP450 enzymes in microbial pharmaceutics had been recognized. However, it was found during application that some enzymes had poor catalytic effect, which could hardly satisfy the current requirement in microbial pharmaceutical field. Promoting coding gene variation was the major route of enzyme modification, which could elevate the sensitivity of enzyme catalysis, and effectively compensate for the drawbacks of natural P450 enzymes. Moreover, it could partly upgrade the enzyme catalytic efficiency.

Bacillus megaterium was a kind of P450 enzymes, which was also the modified enzyme. It had marked advantage in terms of catalytic efficiency compared with naturally occurring P450 enzymes. Some scholars had observed the reaction between narbom ycin and PikC, and compared the values of D50, E85 and E94 in improving catalytic efficiency. The results suggested that, compared with E85 and E94, D50 could more effectively elevate the catalytic yield if it was applied in the catalytic process. E50 was a variant gene, while comparatively speaking, E85 and E94 were wild type genes [2]. It could be found based on the research results that variant gene had more remarkable advantage in catalytic reaction. This had verified the value of modifying CYP450 enzymes in improving catalytic reaction efficiency. 


\section{Drug manufacturing}

Applying CYP450 enzymes in microbial pharmaceutical process, so that they were involved in the catalytic reaction process during pharmaceutics was of positive significance to promote pharmaceutical efficiency and quality. Firstly, P450 enzymes could be applied in drug synthetic process, so that they became the intermediates to promote the effective reaction between the two substances, thus manufacturing the drug. This also accounted for the manufacturing principle of artemisinin. Secondly, the absorption process of drugs in the body was relatively complicated after they entered the body. The other substances should be discharged out of the body through metabolism after the active ingredients were absorbed. CYP450 enzymes were the key substances supporting the realization of the above metabolic process. P450 enzymes could participate in drug screening process to screen out the active ingredients, thus achieving the drug action. Meanwhile, it could act on other substances and remove them out of the body to reduce drug toxicity. Applying them in drug production process could partly promote drug efficacy and safety.

\section{Challenges faced by CYP450 enzymes application}

Progress has been made in the application of CYP450 enzymes in microbial pharmaceutical field. However, the application of P450 enzymes is still challenging due to limitation of factors such as technology and development time. Reducing chaperonin is required in the P450 enzyme catalytic reaction as the major supporting substance. Reducing chaperonin plays a vital role, but it is expensive in price, which is likely to increase the pharmaceutical expenses. In the future, microbial pharmaceutical field should focus on searching for a substitute of reducing chaperonin and applying it into the reaction process, so as to reduce the reaction expenses and further improve the microbial pharmaceutical level.

\section{Conclusion}

In conclusion, greater efforts should be made to optimize and modify CYP450 enzymes, so that they can be applied in microbial drug preparation to boost the overall technical level in microbial pharmaceutical field.

\section{References}

[1] Weis R, Winkler M, Schittmayer M, et al. A Diversified Library of Bacterial and Fungal Bifunctional Cytochrome P450 Enzymes for Drug Metabolite Synthesis[J]. Advanced Synthesis \& Catalysis, 2010, 21(20):2140-2146.

[2] Altaher Y, Kandeel M. Molecular analysis of some camel cytochrome P450 enzymes reveals lower evolution and drug binding properties.[J]. Journal of Biomolecular Structure \& Dynamics, 2016, 34(1):115-124. 